\title{
Role of structural saturation and geometry in the luminescence of silicon-based nanostructured materials
}

\author{
R. Q. Zhang, * J. Costa, and E. Bertran \\ Departament de Fisica Aplicada i Electronica, Universitat de Barcelona, Avenida Diagonal 647, E08028 Barcelona, Spain
}

(Received 1 November 1995)

\begin{abstract}
The structural saturation and stability, the energy gap, and the density of states of a series of small, siliconbased clusters have been studied by means of the PM3 and some ab initio (HF/6-31G* and 6-311++G**, CIS/6-31G* and MP2/6-31G*) calculations. It is shown that in order to maintain a stable nanometric and tetrahedral silicon crystallite and remove the gap states, the saturation atom or species such as $\mathrm{H}, \mathrm{F}, \mathrm{Cl}, \mathrm{OH}, \mathrm{O}$, or $\mathrm{N}$ is necessary, and that both the cluster size and the surface species affect the energetic distribution of the density of states. This research suggests that the visible luminescence in the silicon-based nanostructured material essentially arises from the nanometric and crystalline silicon domains but is affected and protected by the surface species, and we have thus linked most of the proposed mechanisms of luminescence for the porous silicon, e.g., the quantum confinement effect due to the cluster size and the effect of Si-based surface complexes.
\end{abstract}

The visible luminescence in porous silicon ${ }^{1}$ is widely believed to arise from nanometric and crystalline silicon domains which show a quantum confinement effect. ${ }^{1-5}$ However, other studies support different mechanisms of luminescence: several experimental and theoretical papers claimed $^{6-8}$ that both the quantum confinement and the surface (hydrogen saturation) effects are responsible; Brandt et al. ${ }^{9}$ suggested that it may be attributable to a Si-backbone polymer, such as $\mathrm{Si}_{6} \mathrm{O}_{3} \mathrm{H}_{6}$ (some later studies ${ }^{10,11}$ also supported a similar idea); $\mathrm{Xu}, \mathrm{Gal}$, and Gross ${ }^{12}$ assigned luminescence to molecules attached to the Si surface; a few authors attributed it to hydrogen-related surface species such as $\mathrm{SiH}_{2}$ and polysilanes; ${ }^{13,14}$ the formation of amorphous silicon has also been considered as a possible luminescent mechanism. ${ }^{15}$ Some recent papers ${ }^{16-18}$ are very helpful in understanding the origin of the visible luminescence.

The visible luminescence has also been found in other nanoscale silicon-based materials, such as ultrafine silicon particles, ${ }^{19}$ crystallized amorphous $\mathrm{Si}: \mathrm{H} / \mathrm{SiN}_{x}: \mathrm{H}$ multiquantum-well structures, ${ }^{20}$ and the laser-annealed hydrogenated silicon powder produced in plasma-enhanced chemical-vapor deposition processes, ${ }^{21}$ which has recently been shown to be crystallized, ${ }^{22}$ although their photoluminescence (PL) dynamics and energetic distribution are very different. This strongly suggests that the visible luminescence is associated with nanosize silicon-based materials, whose structures can be quite different but must possess local ordering domains. ${ }^{1-8,17-22}$

In this paper, we performed first-principles calculations in order to understand the luminescent mechanism through the study of the atomic and electronic structures of a series of clusters: $\mathrm{Si}_{5}, \mathrm{Si}_{5} X_{12}, \mathrm{Si}_{5} Y_{4}(X=\mathrm{H}, \mathrm{F}, \mathrm{Cl}, \mathrm{OH} ; Y=\mathrm{N})$, $\mathrm{Si}_{17} \mathrm{H}_{n}(n=0,12,24,36), \mathrm{Si}_{29} \mathrm{H}_{36}, \mathrm{Si}_{35} \mathrm{H}_{36}, \mathrm{Si}_{41} \mathrm{H}_{60}$, and $\mathrm{Si}_{17} \mathrm{O}_{12} \mathrm{H}_{12}$. All the ab initio $\left(\mathrm{HF} / 6-31 \mathrm{G}^{*}\right.$ and $6-311++$ $\mathrm{G}^{* *}$, CIS and MP2/6-31G ${ }^{*}$ ) and semiempirical PM3 calculations were carried out with the GAUSSIAN 92 package $^{23}$ on the IBM-sp2 computer at the University of Barcelona. All the structures in this study were optimized; i.e., they were relaxed.

First, we investigated a spherical $\mathrm{Si}_{5}$ cluster with a tetrahedral $T_{d}$ symmetry (two layers of silicon atoms in which a central silicon atom, $\mathrm{Si1}$, is surrounded by four other surface ones, Si2). However, it does not correspond to the global minimum on the energy surface. ${ }^{24}$ In the $T_{d} \mathrm{Si}_{5}$ cluster, the dangling bonds on the surface are almost isolated and form a weak $\pi$ bond with high symmetry around the surface. This highly symmetric, weak $\pi$ bond enables this cluster to be maintained in a substable structure with $T_{d}$ symmetry. From the calculation, we found that there are several danglingbond states inside the gap and it is obvious from the analysis of the density of states ${ }^{25}$ (DOS) that all the gap states come from the surface atoms ( $\mathrm{Si} 2$ ), so these states are localized. The global minimum energy structure, i.e., the most stable structure of the $\mathrm{Si}_{5}$ cluster, is $D 3 h$ symmetry. ${ }^{24}$ For this structure, there is no silicon atom with four coordinates, and the atoms cannot be identified as surface or bulk atoms. The structure is no longer a crystallite, i.e., a crystalline domain. Furthermore, there is no gap state, but a similar local density of states (LDOS) was obtained for each atom.

However, saturation of the dangling bonds of the $T_{d} \mathrm{Si}_{5}$ cluster with 12 hydrogen atoms led to a stable cluster, spherical $\mathrm{Si}_{5} \mathrm{H}_{12}$, with $T_{d}$ symmetry. This cluster may be considered as a crystallite and all the dangling-bond states had moved to the valence and conduction bands. The energy gap obtained is larger than the corresponding one of the $T_{d} \mathrm{Si}_{5}$ as shown in Table I, since the saturation with hydrogen on the surface also removes the influence of dangling bonds on the bulk silicon atoms. It should be mentioned that for a larger unsaturated silicon cluster, the dangling bond may affect several layers of atoms and lead to deviations of the bonds from the bulk ones. In that case, some tail states which correspond to the deviated bonds may appear ${ }^{26}$ and the energy gap seems smaller than that found after hydrogen saturation. On the other hand, the fact that the gap of spherical $T_{d} \mathrm{Si}_{5}$ or $\mathrm{Si}_{5} \mathrm{H}_{12}$, which corresponds to a crystallite, is much larger than the one of stable $D 3 h \mathrm{Si}_{5}$, which was believed to be one kind of amorphous network, implies that if the crystal- 
TABLE I. Summary of some calculated energy gaps (eV).

\begin{tabular}{|c|c|c|c|c|c|c|c|}
\hline \multirow[b]{2}{*}{ Cluster } & \multirow[b]{2}{*}{ Symmetry } & \multirow[b]{2}{*}{$\mathrm{DF}^{\mathrm{a}}$} & \multirow[b]{2}{*}{ PM3 } & \multicolumn{2}{|c|}{ Hartree-Fock } & \multirow{2}{*}{$\begin{array}{c}\text { MP2 } \\
6-31 \mathrm{G}^{*}\end{array}$} & \multirow{2}{*}{$\begin{array}{c}\text { CIS } \\
6-31 G^{*}\end{array}$} \\
\hline & & & & $6-31 G^{*}$ & $6-311++\mathrm{G}^{* *}$ & & \\
\hline $\mathrm{Si}_{5} \mathrm{~b}$ & $T_{d}$ & & 7.14 & 12.64 & & & \\
\hline $\mathrm{Si}_{5}$ & $D 3 h$ & & 4.82 & 7.74 & & & \\
\hline $\mathrm{Si}_{5} \mathrm{H}_{12}$ & $C 2_{v}$ & $5.5(5.8)$ & 6.83 & 12.25 & 11.02 & 12.35 & 10.83 \\
\hline $\mathrm{Si}_{5} \mathrm{H}_{12}$ & $T_{d}$ & 6.3 & 7.28 & 13.44 & 11.57 & 13.47 & \\
\hline $\mathrm{Si}_{17} \mathrm{H}_{36}$ & $T_{d}$ & 5.2 & 6.09 & 11.32 & & & \\
\hline $\mathrm{Si}_{29} \mathrm{H}_{36}$ & $T_{d}$ & 4.9 & 5.59 & 10.58 & & & \\
\hline $\mathrm{Si}_{35} \mathrm{H}_{36}$ & $T_{d}$ & 4.8 & 5.41 & & & & \\
\hline $\mathrm{Si}_{41} \mathrm{H}_{60}$ & $T_{d}$ & & 5.29 & & & & \\
\hline
\end{tabular}

${ }^{\mathrm{a}}$ Data of density-functional approach with self-energy corrections were estimated from Ref. 4, and the datum in the parentheses is experimental; also cited in Ref. 4.

${ }^{\mathrm{b}} \mathrm{A}$ substable structure; see text.

lite produces a visible luminescence the stable noncrystalline network might give a luminescence that is outside the visible region. No experimental evidence was found for visible luminescence in $a-\mathrm{Si}: \mathrm{H}$ or other silicon-based nanosize materials, whose structure was proved to possess no crystalline domains.

We proceeded to investigate the structural stability and saturation effect of a larger cluster, $T_{d}$ symmetry $\mathrm{Si}_{17}$, which may be formed by the $T_{d} \mathrm{Si}_{5}$ with 12 additional silicon atoms in the third layer. For this cluster, there are two types of dangling bond: 12 dangling bonds are isolated and the other 24 may form 12 weak-bent bonds, ${ }^{26}$ as indicated in Fig. 1. If we do not saturate the dangling bonds, we cannot obtain a tetrahedrally $T_{d}$ symmetrical crystallite. As described for the $\mathrm{Si}_{5}$ cluster, the saturation of all 36 dangling bonds for the $\mathrm{Si}_{17}$ cluster with hydrogen atoms also produced a stable structure with $T_{d}$ symmetry. Furthermore, if we saturated only the 24 dangling bonds which may form weak-bent bonds and left the other 12 isolated, the calculation did not converge to a $T_{d}$ crystallite. However, with the saturation of only the 12 isolated dangling bonds, the structure was easily kept in a $T_{d}$ symmetrical crsytallite and the convergence was easily achieved in the calculation. These results indicate that a key factor in obtaining a stable crystallite is the saturation of the isolated dangling bonds.

Certainly these two types, or other similar, of unsaturated dangling bonds can easily be found in a larger unsaturated cluster, and also in the nanostructured materials. Our findings

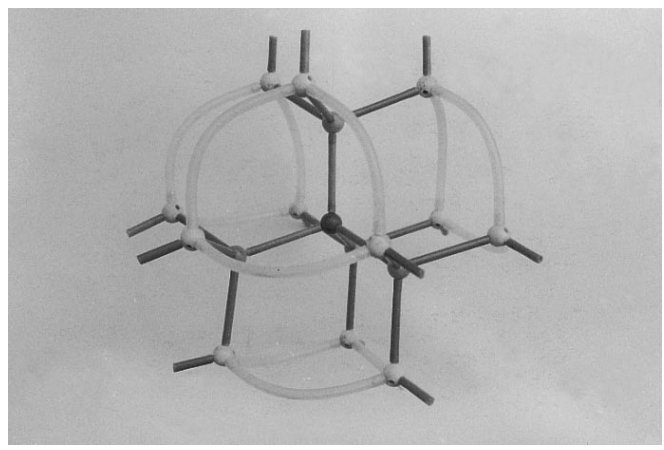

FIG. 1. The structure of the $T_{d} \mathrm{Si}_{17}$ with indications of the isolated dangling bonds (open) and the weak-bent bonds (transparent). suggest that in all cases the role of the isolated dangling bonds in the stability of the structure is the same. We conclude that the cluster with dangling bonds, especially isolated ones, will easily deviate from the crystallite, or in other words, it is difficult to maintain the tetrahedral coordinated structure in the part which is affected by the dangling bonds. To image a practical system, the local structure where the isolated dangling bonds are located may tend to change, which would enable the dangling-bond atoms to form bonds, e.g., weak bond or bent bond, with the others, and thus enhance the stability of the structure. This event may affect several layers of the silicon atoms. If the cluster is large enough, there may still be some crystalline domains surrounded by the noncrystalline network. For these crystallites, the luminescence may be very weak, because of the presence of the noncrystalline layers, which are reflection and absorption centers of light and do not contribute to visible luminescence, but reduce the proportion of the crystallites in the material.

The calculated energy gaps of these clusters and others listed in Table I are much larger than the corresponding value of $1.12 \mathrm{eV}$ of the bulk crystalline silicon, and they are also relatively larger than the gaps given by other theoretical methods. ${ }^{2,4}$ To understand these data, the following three aspects should be noted: (i) the gap becomes smaller when the size of the silicon cluster grows ${ }^{2,4}$ (our calculations in Table I also show this tendency); (ii) the energy gap depends on the basis set and methods used, as shown in the results of the spherical $T_{d}$ and linear $C 2_{v} \mathrm{Si}_{5} \mathrm{H}_{12}$. It seems that inclusion of electron correlation does not lead to a more reasonable energy gap in MP2 calculation, but it gives much improvement in the CIS calculation. We did not perform higher level calculations because of the computational limitation or because it was unnecessary. There is no empirical treatment in the $a b$ initio calculations; thus the results of the structural stability and saturation effect, even the tendency of the energy gap which is based on energy levels, are reliable. ${ }^{27}$ Moreover, although different theoretical methods produced the same tendency of energy gap with the cluster size, the values of the energy gaps for a given cluster are very different. For example, the gap of $\mathrm{Si}_{35}$ is $2.95 \mathrm{eV}$ from a tightbinding method ${ }^{2}$ (TB) and about $4.8 \mathrm{eV}$ from a densityfunctional approach (DF) with a self-energy correction, ${ }^{4}$ 
TABLE II. The calculated energy gaps $(\mathrm{eV})$ for $\mathrm{Si}_{5} X_{12}$ and $\mathrm{Si}_{5} Y_{4}(X=\mathrm{H}, \mathrm{F}, \mathrm{Cl}, \mathrm{OH}$ and $Y=\mathrm{N})$.

\begin{tabular}{lccc}
\hline \hline Cluster & Symmetry & PM3 & HF/6-31G* \\
\hline $\mathrm{Si}_{5} \mathrm{H}_{12}$ & $T_{d}$ & 7.28 & 13.44 \\
$\mathrm{Si}_{5} \mathrm{~F}_{12}$ & $T_{d}$ & 7.09 & 15.19 \\
$\mathrm{Si}_{5} \mathrm{Cl}_{12}$ & $T_{d}$ & 6.14 & 12.98 \\
$\mathrm{Si}_{5} \mathrm{O}_{12} \mathrm{H}_{12}$ & $T_{d}$ & 6.51 & 13.13 \\
$\mathrm{Si}_{5} \mathrm{~N}_{4}$ & $T_{d}$ & 6.13 & 9.11 \\
\hline \hline
\end{tabular}

whereas for a $\mathrm{Si}_{47}$ cluster the TB gave a gap of $2.78 \mathrm{eV}$ and DF with the correction produced a gap of about $4.5 \mathrm{eV}$. Our semiempirical PM3 calculations overestimated the gaps by about $0.6-1.3 \mathrm{eV}$ from the ones given by DF with selfenergy corrections (Table I). (iii) The geometrical optimization of the cluster structure actually enlarges the energy gap, and all the structures in this paper were optimized. An unoptimized geometry is responsible for the tail states of the bands which may narrow the calculated gap.

Moreover, other atoms such as $\mathrm{F}, \mathrm{Cl}$, and $\mathrm{N}$ or species $(\mathrm{OH})$, which may be present in the air, solutions, or other containments, were tested separately as the saturator of the boundary dangling bonds for the $T_{d} \mathrm{Si}_{5}$ cluster. We used one $\mathrm{F}, \mathrm{Cl}$ atom or one $\mathrm{OH}$ group to saturate one dangling bond, whereas one $\mathrm{N}$ atom saturated three dangling bonds. In all cases, similar effects to those obtained with hydrogen as a saturator were found. Only the detailed distribution of the band states and the values of the gap which are shown in Table II are different. The differences of the gaps and the band distributions imply that different saturations produce different distributions of states for a crystallite, and thus the luminescence.

We could not use the oxygen atom to saturate the boundary of the $T_{d} \mathrm{Si}_{5}$ cluster, since it would prefer a site near two dangling bonds. However, it was possible to do this with a $T_{d} \mathrm{Si}_{17}$ cluster where 12 oxygen atoms were used to saturate the 24 dangling bonds which can form 12 bent bonds, and 12 hydrogen atoms saturated the 12 isolated dangling bands. The energy gap was again found to be $5.18 \mathrm{eV}$ by PM3. Similar effects were obtained. From this calculation it was concluded that the oxygen atom is also an efficient saturator for luminescence, which may play an important role in hightemperature processes, e.g., annealing and fabrication.
The cluster models in this study were limited to small size because of the scope of the present calculations, although the luminescence could come from larger domains. However, by considering the importance of the local structural ordering on the luminescence, and that the structural saturation effect and the tendencies of the gaps and density of states do not depend on the cluster size, the conclusions can be reasonably applied to practical silicon-based nanometric materials.

A few previous experiments seem to disagree with the saturation effect in this work. For instance, in the Raman measurements ${ }^{28}$ the UV process and heating to $200{ }^{\circ} \mathrm{C}$ applied to the porous silicon samples in oxygen atmosphere led to amorphous phases saturated with $\mathrm{Si}-\mathrm{O}-\mathrm{H}$ species, accompanied by decreases in the photoluminescence. To understand this, one should notice that the UV or heating process might lead the structure to the amorphous phase along with some $\mathrm{Si}-\mathrm{H}$ bonds being broken. And then some of the dangling bonds produced in the processes could be saturated by $\mathrm{OH}$ species. However, the saturation of dangling bonds could not convert an amorphous network into a crystallite without some processes such as laser and high-temperature annealing together with some saturation atoms, in general. We attribute the PL induced by high-temperature annealing from $a-\mathrm{SiO}$ ${ }_{x}: \mathrm{H} \mathrm{film}^{29}$ to the structure crystallization with the help of oxygen. Moreover, the decreases in PL with thermal annealings at lower temperature in vacuum ${ }^{11,13}$ arose from the structural disordering along with broken $\mathrm{Si}-\mathrm{H}$ bonds. Finally, we could understand most of the reported luminescent phenomena in various silicon-based nanometric materials with the help of the present study.

We have demonstrated the need to saturate the dangling bonds, especially the isolated dangling bonds of Si clusters, to maintain the nanometric and crystalline structural domains, which have been shown to be indispensable in order to produce visible luminescence. The calculation of the energy gap and DOS revealed that both the saturation atoms and the cluster size are responsible for the different luminescence distributions. This research has linked most of the reported mechanisms of the luminescence in porous silicon, such as the confinement effect and surface species effect. Furthermore, we believe this result is useful to understand the luminescence in any silicon-based nanostructured material.

R.Q.Z. is very grateful to Professor W.L. Wang and Professor P. Roura for helpful discussions and to the D.G.I.C.Y.T. for financial support.
*Permanent address: Institute of Optoelectronic Materials and Devices, Shandong University, Jinan, Shandong 250100, P.R. China.

${ }^{1}$ L. T. Canham, Appl. Phys. Lett. 57, 1046 (1990).

${ }^{2}$ X. Wang, D. Huang, L. Ye, M. Yang, P. Hao, H. Fu, X. Hou, and X. Xie, Phys. Rev. Lett. 71, 1265 (1993).

${ }^{3}$ S. Schuppler, S. L. Friedman, M. A. Marcus, D. L. Adler, Y.-H. Xie, F. M. Ross, T. D. Harris, W. L. Brown, Y. J. Chabal, L. E. Brus, and P. H. Citrin, Phys. Rev. Lett. 72, 2648 (1994).

${ }^{4}$ B. Delley and E. F. Steigmeier, Phys. Rev. B 47, 1397 (1993), and references therein.

${ }^{5}$ A. J. Read, R. J. Needs, K. J. Nash, L. T. Canham, P. D. J. Calcott, and A. Qteish, Phys. Rev. Lett. 69, 1232 (1992).

${ }^{6}$ K.-H. Li, C. Tsai, S. Shih, T. Hsu, D. L. Kwong, and J. C. Camp- bell, J. Appl. Phys. 72, 3816 (1992).

${ }^{7}$ M. B. Robinson, A. C. Dillon, and S. M. George, Appl. Phys. Lett. 62, 1493 (1993).

${ }^{8}$ S.-G. Lee, B.-H. Cheong, K.-H. Lee, and K. J. Chang, Phys. Rev. B 51, 1762 (1995).

${ }^{9}$ M. S. Brandt, H. D. Fuchs, M. Stutzmann, J. Weber, and M. Cardona, Solid State Commun. 81, 307 (1992).

${ }^{10}$ J. M. Lavine, S. P. Sawan, Y. T. Shieh, and A. J. Bellezza, Appl. Phys. Lett. 62, 1099 (1993).

${ }^{11}$ Y. Xiao, M. J. Heben, J. M. McCullough, Y. S. Tsuo, J. I. Pankove, and S. K. Deb, Appl. Phys. Lett. 62, 1152 (1993).

${ }^{12}$ Z. Y. Xu, M. Gal, and M. Gross, Appl. Phys. Lett. 60, 1375 (1992).

${ }^{13}$ C. Tsai, K.-H. Li, J. Sarathy, S. Shih, J. C. Campbell, B. K. 
Hance, and J. M. White, Appl. Phys. Lett. 59, 2814 (1992).

${ }^{14}$ C. Tsai, K.-H. Li, D. S. Kinosky, R.-Z. Qian, T. C. Hsu, J. T. Irby, S. K. Banerjee, A. F. Tasch, J. C. Campbell, B. K. Hance, and J. M. White, Appl. Phys. Lett. 60, 1700 (1992).

${ }^{15}$ R. P. Vasquez, R. W. Fathauer, T. George, A. Ksendzov, and T. L. Lin, Appl. Phys. Lett. 60, 1004 (1992).

${ }^{16}$ S. M. Prokes, W. E. Carlos, and O. J. Glembocki, Phys. Rev. B 50, 17093 (1994).

${ }^{17}$ S. Schuppler, S. L. Friedman, M. A. Marcus, D. L. Adler, Y.-H. Xie, F. M. Ross, Y. J. Chabal, T. D. Harris, L. E. Brus, W. L. Brown, E. E. Chaban, P. F. Szajowski, S. B. Christman, and P. H. Citrin, Phys. Rev. B 52, 4910 (1995).

${ }^{18}$ L. E. Brus, P. F. Szajowski, W. L. Wilson, T. D. Harris, S. Schuppler, and P. H. Citrin, J. Am. Chem. Soc. 117, 2915 (1995).

${ }^{19}$ S. Furukawa and T. Miyasato, Superlattices Microstruct. 5, 317 (1989); H. Morisaki, F. W. Ping, H. Ono, and K. Yazawa, J. Appl. Phys. 70, 1869 (1991).

${ }^{20}$ K. J. Chen, X. F. Huang, J. Xu, and D. Feng, Appl. Phys. Lett. 61,
2069 (1992).

${ }^{21}$ J. Costa, P. Roura, G. Sardin, J. R. Morante, and E. Bertran, Appl. Phys. Lett. 64, 463 (1994); P. Roura, J. Costa, G. Sardin, J. R. Morante, and E. Bertran, Phys. Rev. B 50, 18124 (1994).

${ }^{22}$ J. Costa, P. Roura, A. Canillas, E. Pascual, J. R. Morante, and E. Bertran (unpublished).

${ }^{23}$ M. J. Frisch et al., Gaussian 92, Revision A (Gaussian, Inc., Pittsburgh, PA, 1992).

${ }^{24}$ K. Raghavachari, J. Chem. Phys. 84, 5672 (1986).

${ }^{25}$ R. Q. Zhang, J. J. Wang, G. C. Dai, J. A. Wu, J. P. Zhang, and Y. R. Xing, Chin. J. Semicond. 10, 327 (1989).

${ }^{26}$ R. Zhang, Solid State Commun. 69, 681 (1989).

${ }^{27}$ W. J. Hehre, L. Radom, P. v. R. Schleyer, and J. A. Pople, $A b$ initio Molecular Orbital Theory (Wiley, New York, 1986).

${ }^{28}$ J. C. Tsang, M. A. Tischer, and R. T. Collins, Appl. Phys. Lett. 60, 2279 (1992).

${ }^{29}$ C.-H. Lin, S.-C. Lee, and Y.-F. Chen, Appl. Phys. Lett. 63, 902 (1993). 